\title{
Green synthesis and characterization of silver nanoparticles using Artemisia absinthium aqueous extract- A comprehensive study
}

\author{
Mohammad Ali ${ }^{1}$, Bosung Kim², Kevin Belfield ${ }^{2,3}$, David Norman', Mary Brennan ${ }^{1}$ \\ and Gul Shad Ali ${ }^{1^{*}}$ \\ ${ }^{1}$ Mid-Florida Research and Education Center and Department of Plant Pathology, \\ University of Florida/Institute of Food and Agricultural Sciences, 2725 Binion Rd, \\ Apopka, FL 32703 \\ ${ }^{2}$ Department of Chemistry, University of Central Florida, Orlando, FL 32816 \\ ${ }^{3}$ College of Science and Liberal Arts, New Jersey Institute of Technology, Newark, NJ \\ 07102
}

*Corresponding author: Gul Shad Ali;

Email address: gsali@ufl.edu

Tel:+1-407-410-6933

Fax:+1-407814-6186

(C) 2015. This manuscript version is made available under the Elsevier user license http://www.elsevier.com/open-access/userlicense/1.0/ 


\begin{abstract}
Unlike chemical synthesis, biological synthesis of nanoparticles is gaining tremendous interest, and plant extracts are preferred over other biological sources due to their ample availability and wide array of reducing metabolites. In this project, we investigated the reducing potential of aqueous extract of Artemisia absinthium L. for synthesizing silver nanoparticles (AgNPs). Optimal synthesis of AgNPs with desirable physical and biological properties was investigated using ultra violet-visible spectroscopy (UV-vis), dynamic light scattering (DLS), transmission electron microscopy (TEM) and energydispersive X-ray analysis (EDX). To determine their appropriate concentrations for AgNPs synthesis, two-fold dilutions of silver nitrate (20 to $0.62 \mathrm{mM}$ ) and aqueous plant extract (100 to $\left.0.79 \mathrm{mg} \mathrm{mL}^{-1}\right)$ were reacted. The results showed that silver nitrate $(2 \mathrm{mM})$ and plant extract $\left(10 \mathrm{mg} \mathrm{mL}^{-1}\right)$ mixed in different ratios significantly affected size, stability and yield of $\mathrm{AgNPs}$. Extract to $\mathrm{AgNO}_{3}$ ratio of $6: 4 \mathrm{v} / v$ resulted in highest conversion efficiency of $\mathrm{AgNO}_{3}$ to AgNPs, with the particles in average size range of less than $100 \mathrm{~nm}$. Furthermore, the direct imagining of synthesized AgNPs by TEM revealed polydispersed particles in the size range of 5 to $20 \mathrm{~nm}$. Similarly, nanoparticles with the characteristic peak of silver were observed with EDX. This study presents a comprehensive investigation of the differential behavior of plant extract and $\mathrm{AgNO}_{3}$ to synthesize biologically stable AgNPs.
\end{abstract}

Key words: AgNPs, DLS, EDX, Artemisia

\title{
1. Introduction
}

Nanoparticle synthesis and characterization is an area of immense interest due to its broader applicability in the fields of medicine, biology, physics and chemistry [1]. Silver nanoparticles (AgNPs) are gaining particular attention due to their desirable properties, including their high surface to volume ratios, catalytic properties and antimicrobial effects [2]. However, most techniques used for the synthesis of nanoparticles are expensive and may adversely affect the environment and biological systems. Green synthesis, on the other hand, offers comparatively safer and eco-friendly approach for nanoparticles synthesis. During the last five years, attempts have been made to develop 
various greener and cheaper strategies for nanoparticles biosynthesis (Rauwel et al. 2015). Plants provide a highly desirable system for nanoparticles synthesis due to their ability to produce a wide range of secondary metabolites with strong reducing potential. Since plants are less sensitive to metal toxicity compared to algae and bacteria, they offer a green alternative for the biosynthesis of AgNPs [3]. Medicinal plants are of special concern since they control the size and shape of nanoparticles by providing capping layers to nanoparticles (Rauwel et al. 2015).

Plant secondary products provide rich resources as potential drugs, nutraceuticals, and food additives. Plant polyphenols are one of the largest groups of natural antioxidant secondary metabolites [4]. The reduction properties of these antioxidant metabolites can be linked with the higher potential ability of plant extracts to synthesize nanoparticles with improved characteristics [5]. The genus Artemisia in the Asteraceae family consists of many diverse species that produce many medicinally valuable secondary metabolites including the widely used antimalarial drug artemisinin [6]. Artemisia absinthium is an important medicinal herb, naturally found in the foothills of Himalayas in the Indian subcontinent. Due to its health protective effects, this species was considered a general remedy for many diseases [7]. Various biological activities attributed to this herb include neuroprotective [8,9], hepatoprotective [10, 11], anticancer [12], antiplasmodial [13, 14] and antioxidant [15-17]. The strong antioxidant activity and the biosynthetic potential of this species for phenolics and flavonoids [18-21] may be considered as potential factors for reducing $\mathrm{Ag}^{+}$to AgNPs. Previously, biological synthesis of AgNPs by using $A$. pallens [22], A. capillaris [23] and A. annua [24] was reported. However, to the best of our knowledge, there are no reports aimed to synthesize AgNPs by using A. absinthium as biological substrate. Therefore, as a novel study, the present work was aimed to evaluate the differential potential of $A$. absinthium aqueous extract and silver nitrate to synthesize biologically stable silver nanoparticles.

\section{Materials and Methods}

\subsection{Plant material and preparation of extract}


Green house-raised A. absinthium L. plants were dried at room temperature and used for AgNPs synthesis. Plant extract was prepared by boiling 100 to $1000 \mathrm{mg}$ of the dried powdered leaves of $A$. absinthium in $10 \mathrm{ml}$ deionized water for 5 minutes. The aqueous extract was cooled down to room temperature $\left(25^{\circ} \mathrm{C}\right)$, filtered through a 0.45 um filter $\left(\right.$ Millex $^{\circledR}$ ) and stored at $4^{\circ} \mathrm{C}$ until used for further analysis.

\subsection{Synthesis of silver nanoparticles}

For the synthesis of silver nanoparticles, $\mathrm{AgNO}_{3}(2 \mathrm{mM})$ and aqueous plant extract (10 $\mathrm{mg} \mathrm{mL}^{-1}$ ) were mixed in different ratios. Briefly, $100 \mu \mathrm{l}$ of plant extract was mixed with $900 \mu \mathrm{l}$ of $\mathrm{AgNO}_{3}$ (1:9 ratio). The subsequent mixtures were prepared by increasing plant extract and decreasing $\mathrm{AgNO}_{3}$ volumes by $100 \mu$, until the final ratio of 9:1 was attained. Furthermore, appropriate concentrations of $\mathrm{AgNO}_{3}$ and plant extract for optimal AgNPs synthesis were determined in a series of reactions, containing mixtures of equal volumes of two-fold serial dilutions of $\mathrm{AgNO}_{3}$ (20 to $0.62 \mathrm{mM}$ ) and plant extract (100 to $0.79 \mathrm{mg}$ $\left.\mathrm{mL}^{-1}\right)$. These mixtures were prepared either in 1.5 -mL Eppendorf tubes by mixing $500 \mu 1$ of each reactant or in 96-well microtiter plates (cat\# CC7672-7596, www.CytoOne.com) by mixing $100 \mu \mathrm{l}$ of each reactant. The reactions were allowed to progress at room temperature for different time periods.

\subsection{UV-vis spectrometry and Dynamic Light Scattering (DLS)}

AgNPs synthesis was monitored by recording UV-vis spectra $(\lambda 300-600 \mathrm{~nm}) \mathrm{using}$ either a NanoDrop 2000C Spectrophotometer (Thermo Fischer Scientific, USA) or the Synergy H1 Hybrid multi-mode microplate reader $\left(\right.$ BioTek $\left.^{\circledR}\right)$. Unreacted $\mathrm{AgNO}_{3}$ and plant extracts were removed by pelleting and washing AgNPs as follows. One mL of each reaction mixture was centrifuged at $14,000 \mathrm{xg}$ for 10 minutes at room temperature. Supernatants were discarded and the AgNPs pellets were resuspended in one $\mathrm{mL}$ deionized water followed by centrifugation at 14,000xg for 10 minutes. This process was repeated five times. The resulting AgNPs were resuspended in deionized water, and used for characterization. The particle size and $\zeta$-potential of all samples were measured by pipetting one $\mathrm{mL}$ of each AgNP sample in UV-vis DLS cuvettes and were analyzed with Zetasizer Nano-ZS90 (Malvern Instruments). The $\zeta$-potential was automatically calculated by determining electrophoretic velocity. 


\subsection{Transmission Electron Microscopy (TEM) and Energy Dispersive X-ray (EDX)}

For TEM and EDX analyses, $2 \mu \mathrm{l}$ aqueous suspensions of AgNPs were pipetted onto 400 mesh carbon-coated copper grids. Samples were allowed to evaporate and dry in a hood. TEM images were collected with FEI Technai F30 transmission electron microscope. Elemental analyses of the AgNPs were performed using the same Technai F30 TEM using an Energy Dispersive X-ray (EDX) detector.

\section{5. $\mathrm{Ag}^{+}$to AgNPs Conversion efficiency calculation}

Conversion efficiency of $\mathrm{AgNO}_{3}$ to AgNPs by plant extract was determined as follows. Ten mL AgNPs suspensions were washed five times as described above. Washed pellets were completely dried in a speed-vac and weighed. Percent conversion efficiency (CE) was calculated using the following formula:

$C E=(A g N P s / A g) \times 100$, where "AgNPs" is the output weight of AgNPs formed and " $A g$ " is the input weight of $\mathrm{Ag}^{+}$ions.

\section{Results}

\subsection{Synthesis kinetics and characterization of AgNPs by UV-vis Spectroscopy}

The change in the color of reaction mixture to yellowish brown or dark brown after mixing a plant extract and silver nitrate is a general characteristic of silver nanoparticles biosynthesis. In our initial experiments assaying A. absinthium aqueous extract with $\mathrm{AgNO}_{3}$, reaction mixtures started turning brown within 30 minutes and it continued turning darker with time (Figure 1a). The reaction mixture with 1:1 $(v / v)$ ratio of each reactant turned darker more rapidly, compared to the rest of reaction mixtures. However, no color change was observed with the plant extract or $\mathrm{AgNO}_{3}$ alone under the same conditions.

Next, the effect of relative ratios of plant extract and $\mathrm{AgNO}_{3}$ on $\mathrm{AgNPs}$ synthesis was analyzed using UV-visible spectroscopy. In preliminary experiments, when mixed in a $1: 1(v / v)$ ratio, the plant extract and $\mathrm{AgNO}_{3}$ showed an increase in UV-vis spectrum above $350 \mathrm{~nm}$, with the most pronounced increase in the 400 to $500 \mathrm{~nm}$ range (Figure 
1b). These analyses showed different intensities of surface plasmon resonance peaks in the range of 435-445 $\mathrm{nm}$ with different plant extract: $\mathrm{AgNO}_{3}$ ratios. In general, intensities of absorbance maximums increased with an increase in plant extract from 10 to $50 \%$, whereas absorbance maximums decreased with a decrease in plant extract from 60 to $10 \%$. Highest absorbance with comparatively narrow peak was observed when both reactants were used in 1:1 ( v/v) ratio. However, the mixture with 9:1 $(v / v)$ ratio of plant extract and $\mathrm{AgNO}_{3}$ did not show the characteristic peak for silver nanoparticles.

Time-course analysis of UV-vis data of plant extract: $\mathrm{AgNO}_{3}(1: 1 \mathrm{v} / \mathrm{v})$ mixture showed accumulation of AgNPs within an hour of the reaction start time (Figure 2a). Typically, AgNPs in colloidal solutions display maximum absorption in the $\lambda 400-500$ $\mathrm{nm}$ range. To capture yield of most AgNPs in this range, statistical analyses were performed with the sum of $\lambda 400-500 \mathrm{~nm}$. A scatter plot of the time-response UV-vis data indicated that AgNPs synthesis exhibited typical chemical kinetics. Curve fitting revealed that AgNPs synthesis fitted an exponential model with excellent goodness-of-fit parameters $\left(\mathrm{R}^{2}=0.96, \mathrm{Sy} \cdot \mathrm{x}=0.61\right)$. AgNPs synthesis increased exponentially with a time constant $(\tau)$ of 4.13 hours (3.17 to 5.9, 95\% confidence intervals) and a half life of 2.86 hours (2.1 to 4.1, 95\% confidence intervals) (Figure 2b and Figure S1). AgNPs synthesis leveled off at 24 hours after the start of reaction with little or no increase afterwards.

\subsection{Titration analysis for optimum synthesis of AgNPs}

To determine an appropriate concentration of $\mathrm{AgNO}_{3}$ for optimal AgNPs synthesis, a range of $\mathrm{AgNO}_{3}$ concentrations (512 to $0.0156 \mathrm{mM}$ ) were tested. Bar graph plots indicated that dose-response $\left(\mathrm{AgNO}_{3}\right.$ concentration-AgNPs synthesis) data formed a bellshaped curve. Indeed, the data fitted a bell-shaped curve with excellent goodness-of-fit parameters $\left(\mathrm{R}^{2}=0.98\right.$, and Sy.x $\left.=0.57\right)$ (Figure 3a). From the bell-shaped curve fits, we could calculate that $\mathrm{AgNO}_{3}$ in the range of $2 \mathrm{mM}$ to $16 \mathrm{mM}$ resulted in optimum $\mathrm{AgNPs}$ synthesis. Optimal average AgNPs yields of 96.7, 122.7, 120.6, 104.1 $\mu \mathrm{g} \mathrm{mL}^{-1}$ were obtained with 2, 4, 8 and $16 \mathrm{mM} \mathrm{AgNO}_{3}$, respectively. Similarly, in this concentration range, $\mathrm{Ag}^{+}$to $\mathrm{AgNPs}$ conversion efficiencies (AgNPs output/ $\mathrm{Ag}^{+}$input) were also higher, reaching approximately $90 \%$ with $2 \mathrm{mM} \mathrm{AgNO}_{3}$ (Figure 3b). Next, we investigated the effect of the concentration of plant extract in the reaction mixture on AgNPs synthesis at 
constant $\mathrm{AgNO}_{3}$ concentrations ( 1 and $2 \mathrm{mM}$ ). Similar to the effect of $\mathrm{AgNO}_{3}$, doseresponse data with plant extract also fitted bell-shaped curves, indicating that AgNPs synthesis is optimal at intermediate levels of plant extracts (Figure $3 \mathrm{c}, 3 \mathrm{e}$ and $3 \mathrm{~g}$ ). Similarly, conversion efficiencies with different concentrations of plant extract also followed bell-shaped curves (Figure $3 \mathrm{~d}, 3 \mathrm{f}$ and $3 \mathrm{~h}$ ). These analyses indicate that optimal AgNPs synthesis occurs at specific ratios of plant extract and $\mathrm{AgNO}_{3}$. These results were further verified in a $2 \times 2$ factorial experiment, which consisted of 6 and 8 two-fold serial dilutions of $\mathrm{AgNO}_{3}\left(20\right.$ to $0.62 \mathrm{mM}$ ) and plant extract (100 to $0.79 \mathrm{mg} \mathrm{mL}^{-1}$ ), respectively. Visual examination of the reaction mixtures at different time points after the start of reactions showed that at higher plant extract and $\mathrm{AgNO}_{3}$ concentrations, AgNPs agglomerated and sedimented instead of staying in colloidal state (Figure S2a). UV-vis data of these experiments also showed that optimal AgNPs synthesis is dependent on the relative concentrations of plant extract and $\mathrm{AgNO}_{3}$ (Figure $\mathrm{S} 2 \mathrm{~b}$ ).

\subsection{Size distribution and $\zeta$-potential of AgNPs}

Hydrodynamic size distribution of AgNPs was analyzed using dynamic light scattering (DLS). DLS analysis revealed a wider hydrodynamic size range, approximately 5 to 200 $\mathrm{nm}$ radius, with different ratios of plant extract and $\mathrm{AgNO}_{3}$ (Figure $4 \mathrm{a}-\mathrm{h}$, left panel). In general, the average hydrodynamic sizes of AgNPs increased with increasing plant extract concentration (Table 1).

Absolute $\zeta$-potential values measure stability of nanoparticles in suspension form.

In this study, $\zeta$ - potential of AgNPs synthesized with different plant extract: $\mathrm{AgNO}_{3}$ ratios were evaluated. $\zeta$-potentials ranged from -32.4 and $-40.4 \mathrm{mV}$. With plant extract: $\mathrm{AgNO}_{3}$ ratios of 4:6, 5:5, 6:4, 7:3 and 8:2, absolute $\zeta$-potential values decreased, which were $40.4 \mathrm{mV},-34.6 \mathrm{mV},-34.5 \mathrm{mV}$ and $-32.4 \mathrm{mV}$, respectively (Figure 4i-p, right panel). However, at plant extract: $\mathrm{AgNO}_{3}$ ratios of 1:9, 2:8 and 3: 7, $\zeta$ - potential values were $39.6 \mathrm{mV},-39.8 \mathrm{mV}$ and $-38.9 \mathrm{mV}$, indicating that plant extract: $\mathrm{AgNO}_{3}$ ratios do not affect $\zeta$-potentials substantially.

\subsection{TEM and EDX analyses of AgNPs}

The shapes and sizes of AgNPs were analyzed using TEM. Multiple TEM images revealed different shapes and sizes of AgNPs with most in the range of 5 to $20 \mathrm{~nm}$ 
diameter. Although, most particles did not agglomerate, several particles appeared to be enrobed in irregularly shaped substances (Figure 5a, arrows). High-resolution TEM images showed characteristic lattice fringes and fringe width $(0.23 \mathrm{~nm})$ of $\mathrm{Ag}$ (Figure 5b). Furthermore, these images also showed that most AgNPs were round shaped and polydispersed. Most particles displayed multiple-twinned crystalline planes, indicating that they consist of several differentially oriented crystals (Figure 5b).

Chemical composition of AgNPs was determined using local EDX micro analysis of several individual particles. EDX spectra revealed major energy peaks corresponding to $\mathrm{Ag}, \mathrm{N}, \mathrm{C}$ and $\mathrm{Cu}$ (Figure 5c). Several peaks were observed around the Ag main peak indicative of different valency states of $\mathrm{Ag}$ in the particles. Peaks corresponding to $\mathrm{Cu}$ and $\mathrm{C}$ are routinely observed in samples deposited on $\mathrm{C}$-coated copper grids.

\section{Discussion}

Due to its nontoxic nature for human use, silver metal has been utilized for various purposes since a long time [25]. AgNPs synthesized using biological substrates are used in different areas of basic and applied research. Recent reports have demonstrated that AgNPs produced with plant extract have excellent antibacterial, antioxidant and cytotoxicity activities [26-28]. In this study, we showed that $A$. absinthium plant extract has strong potential for synthesizing AgNPs, which will likely display antibacterial activity. The reduction of $\mathrm{AgNO}_{3}$ in the presence of antioxidant metabolites, phenolics and flavonoids results in a characteristic shift in surface plasmon resonance, which indirectly indicates the presence of AgNPs [29-31]. In general, peaks at longer wavelengths indicate an increase in particle size, whereas peaks at shorter wavelengths represent smaller particle size $[32,33]$. Consistent with these observations, we observed absorption in the shorter spectrum from $380-450 \mathrm{~nm}$, depending on the shape and size of the NPs. Similarly, spectrum width at half maximum of a peak was also broad (Figure 1 and Figure S1) suggesting that AgNPs synthesized in this study are polydispersed. This notion was supported by mixed populations of particles observed in several independent TEM images (Figure 5). Polydispersity may be the result of tremendous variability of 
various secondary metabolites with potentially different reduction properties, which may affect AgNPs nucleation and growth.

Size and charge are very important parameters for the bioactive properties and stability of nanoparticles. Titration assays showed that synthesis of AgNPs that were very well dispersed in colloidal solutions with characteristic UV-vis spectra required appropriate relative concentrations of $A$. absinthium extract and $\mathrm{AgNO}_{3}$. These results are further supported by the bell-shaped curves of dose-response data in Figure 3, which are consistent with reactions that are dependent on relative concentrations of reactants. Mechanistically, these results indicated that the reaction of $\mathrm{Ag}^{+}$with an unknown plant metabolite(s) may not be a simple redox reaction between two compounds, but it might also involve a protein catalyst with binding sites for $\mathrm{Ag}^{+}$and plant metabolites. This scenario is reminiscent of some enzymes they are stimulated at lower substrate concentrations but inhibited at higher substrate concentration and therefore follow bellshaped kinetics [34]. Testing this hypothesis, however, would require extensive experimentation in future studies. At higher concentrations of $\mathrm{AgNO}_{3}$ and/or $A$. absinthium extracts, larger clusters of particles formed, which could have resulted due to a very high localized concentration of reducing reagents in the solution. These larger clusters appeared as irregularly shaped precipitates, which were resistant to dispersing into a colloidal solution with vigorous vortexing. They were, however, dispersed into a colloidal solution with sonication, suggesting that they are held together by non-covalent weak forces. DLS analyses showed that the hydrodynamic size (diameter) of the particles increased from $78.2 \mathrm{~nm}$ to $160.6 \mathrm{~nm}$ with an increase in plant extract. The increase in particle size might be due to the agglomeration of particles in response to higher concentration of reducing metabolites in the plant extract. In fact, in TEM images large aggregates of nanoparticles embedded in an amorphous substance were observed (Figure 5). These large substances might be the larger hydrodynamic particles detected in the DLS studies. In previous studies, larger sizes of silver nanoparticles (329 $\mathrm{nm})$ and gold nano particles $(300 \mathrm{~nm})$ determined by DLS were actually due to agglomeration, which were verified in TEM images $[35,36]$. Future studies that focus on determining the chemical composition of amorphous substances could shed light on their origin and role in providing stability to AgNPs. 
Zetasizer studies, aimed at determining surface charge, dispersion, and stability of AgNPs synthesized in this study, revealed that $A$. absinthium extract is well suited for synthesizing AgNPs. Due to the presence of various secondary metabolites, we expected differences in the $\zeta$-potential of AgNPs synthesized with different concentrations of plant extracts. With a decrease in surface plasmon resonance from $440 \mathrm{~nm}$ to $435 \mathrm{~nm}$, the corresponding $\zeta$-potential values increased from $-40.4 \mathrm{mV}$ and $-34.6 \mathrm{mV}$, and corresponding particle sizes increased from $93.8 \mathrm{~nm}$ to $103.40 \mathrm{~nm}$. Since higher absolute $\zeta$-potential values indicate higher NP stability, these results indicate that more stable nanoparticles were formed when the surface plasmon resonance shifted to a greater wavelength. Our results are consistent with a previous study where it was found that for minor changes in the $\zeta$-potential value from -31.42 to $-29.10 \mathrm{mV}$ there was a corresponding notable increase in the hydrodynamic diameter from 108.6 to $144.6 \mathrm{~nm}$ [37]. Also, there was not a change in the UV-vis or $\zeta$-potentials of AgNPs stored at room temperature as colloidal solution in water after at least one year of synthesis, suggesting that AgNPs reported in this study are highly stable.

\section{Conclusions}

In conclusion, in this study we showed that A. absinthium mediated AgNPs biosynthesis is strongly dependent on the relative concentrations of plant extract and $\mathrm{AgNO}_{3}$. The higher concentration of plant extract or $\mathrm{AgNO}_{3}$ resulted in bigger sized nanoparticles which sediment down the gravity due to higher agglomeration.

Furthermore, almost equal volumes of plant extract and $\mathrm{AgNO}_{3}$ resulted in comparatively smaller sized and stable nanoparticles. This study will be helpful as an optimization protocol for biological synthesis of AgNPs by using other medicinal plants.

\section{Acknowledgments}

We are thankful to Mikhail Klimov, University of Central Florida, Advanced Materials Processing and Analysis Center, Materials Characterization Facility for his excellent help with TEM and EDX analyses. This work was supported by funds to G.S.A. from the 
Florida Agriculture Experiment Station, Institute of Food and Agricultural Sciences at the University of Florida. M. A. was supported by a Graduate Student Fellowship by the Higher Education Commission of Pakistan. K.D.B acknowledges support from the National Science Foundation (CHE-0832622).

\section{References}

[1] J.Y. Song, B.S. Kim, Rapid biological synthesis of silver nanoparticles using plant leaf extracts, Bioprocess Biosyst. Eng., 32 (2009) 79-84.

[2] F. Okafor, A. Janen, T. Kukhtareva, V. Edwards, M. Curley, Green synthesis of silver nanoparticles, their characterization, application and antibacterial activity, Int $\mathbf{J}$ Environ Res Public Health, 10 (2013) 5221-5238.

[3] S. Pandey, A. Mewada, M. Thakur, R. Shah, G. Oza, M. Sharon, Biogenic gold nanoparticles as fotillas to fire berberine hydrochloride using folic acid as molecular road map, Mater Sci Eng C Mater Biol Appl, 33 (2013) 3716-3722.

[4] Ł. Cieśla, I. Kowalska, W. Oleszek, A. Stochmal, Free Radical Scavenging Activities of Polyphenolic Compounds Isolated from Medicago sativa and Medicago truncatula Assessed by Means of Thin-layer Chromatography DPPH` Rapid Test, Phytochemical Analysis, 24 (2013) 47-52.

[5] O.V. Kharissova, H.V. Dias, B.I. Kharisov, B.O. Perez, V.M. Perez, The greener synthesis of nanoparticles, Trends Biotechnol., 31 (2013) 240-248.

[6] K.S. Bora, A. Sharma, The genus Artemisia: a comprehensive review, Pharmaceutical Biology, 49 (2011) 101-109.

[7] P. Baker, The book of absinthe: a cultural history, Grove Press2003. 
[8] K.S. Bora, A. Sharma, Neuroprotective effect of Artemisia absinthium L. on focal ischemia and reperfusion-induced cerebral injury, Journal of ethnopharmacology, 129 (2010) 403-409.

[9] Y. Li , Y. Ohizumi, Search for constituents with neurotrophic factor-potentiating activity from the medicinal plants of Paraguay and Thailand, Yakugaku Zasshi, 124 (2004) 417-424.

[10] N. Amat, H. Upur, B. Blažeković, In vivo hepatoprotective activity of the aqueous extract of Artemisia absinthium L. against chemically and immunologically induced liver injuries in mice, Journal of ethnopharmacology, 131 (2010) 478-484.

[11] M. Sharma, S. Shukla, Reversal of Carbon Tetrachloride-Induced Hepatic Injury by Aqueous Extract of Artemisia absinthium in Sprague-Dawley Rats, Journal of Environmental Pathology, Toxicology and Oncology, 31 (2012).

[12] G. Shafi, T.N. Hasan, N.A. Syed, A.A. Al-Hazzani, A.A. Alshatwi, A. Jyothi, A. Munshi, Artemisia absinthium (AA): a novel potential complementary and alternative medicine for breast cancer, Molecular biology reports, 39 (2012) 7373-7379.

[13] A. Ramazani, S. Sardari, S. Zakeri, B. Vaziri, In vitro antiplasmodial and phytochemical study of five Artemisia species from Iran and in vivo activity of two species, Parasitology research, 107 (2010) 593-599.

[14] S. Irshad, A. Mannan, B. Mirza, Antimalarial activity of three Pakistani medicinal plants, Pakistan journal of pharmaceutical sciences, 24 (2011) 589-591.

[15] M. Ali, B.H. Abbasi, Ihsan-ul-Haq, Production of commercially important secondary metabolites and antioxidant activity in cell suspension cultures of Artemisia absinthium L, Industrial Crops and Products, 49 (2013) 400-406.

[16] J.M. Canadanovic-Brunet, S.M. Djilas, G.S. Cetkovic, V.T. Tumbas, Free-radical scavenging activity of wormwood (Artemisia absinthium $\mathrm{L}$ ) extracts, Journal of the Science of Food and Agriculture, 85 (2005) 265-272. 
[17] O. Kharoubi, M. Slimani, D. Krouf, L. Seddick, A. Aoues, Role of wormwood (Artemisia absinthium) extract on oxidative stress in ameliorating lead induced haematotoxicity, African Journal of Traditional, Complementary and Alternative Medicines, 5 (2008) 263-270.

[18] M. Ali, B.H. Abbasi, Light-induced fluctuations in biomass accumulation, secondary metabolites production and antioxidant activity in cell suspension cultures of Artemisia absinthium L, J. Photochem. Photobiol. B, 140 (2014) 223-227.

[19] M. Ali, B.H. Abbasi, Thidiazuron-induced changes in biomass parameters, total phenolic content, and antioxidant activity in callus cultures of Artemisia absinthium L., Appl. Biochem. Biotechnol., 172 (2014) 2363-2376.

[20] M. Ali, B.H. Abbasi, Ihsan-ul-Haq, Production of commercially important secondary metabolites and antioxidant activity in cell suspension cultures of Artemisia absinthium L., Industrial Crops and Products, 49 (2013) 400-406.

[21] Y.-J. Lee, M. Thiruvengadam, I.-M. Chung, P. Nagella, Polyphenol composition and antioxidant activity from the vegetable plant Artemisia absinthium L, AJCS, 7 (2013) 1921-1926.

[22] S.M. Arde, P.R. Salokhe, A.H. Mane, R.S. Salunkhe, Facile Green Synthesis of Silver Nanoparticles by Artemisia pallens Leaves Extract and Evaluation of Antimicrobial Activity, Chem Sci Rev Lett, 3 (2014) 557-562.

[23] Y. Park, H.J. Noh, L. Han, H.-S. Kim, Y.-J. Kim, J.S. Choi, C.-K. Kim, Y.S. Kim, S. Cho, Artemisia capillaris extracts as a green factory for the synthesis of silver nanoparticles with antibacterial activities, Journal of nanoscience and nanotechnology, 12 (2012) 7087-7095.

[24] A. Johnsona, I. Obota, U. Ukponga, Green synthesis of silver nanoparticles using Artemisia annua and Sida acuta leaves extract and their antimicrobial, antioxidant and corrosion inhibition potentials, J. Mater. Environ. Sci, 5 (2014) 899-906. 
[25] J.L. Elechiguerra, J.L. Burt, J.R. Morones, A. Camacho-Bragado, X. Gao, H.H. Lara, M.J. Yacaman, Interaction of silver nanoparticles with HIV-1, J nanobiotechnol, 3 (2005) 1-10.

[26] M.K. Swamy, K. Sudipta, K. Jayanta, S. Balasubramanya, The green synthesis, characterization, and evaluation of the biological activities of silver nanoparticles synthesized from Leptadenia reticulata leaf extract, Applied Nanoscience, 5 (2015) 73 81.

[27] R. Mata, J.R. Nakkala, S.R. Sadras, Catalytic and biological activities of green silver nanoparticles synthesized from Plumeria alba (frangipani) flower extract, Materials Science and Engineering: C, 51 (2015) 216-225.

[28] J.R. Nakkala, R. Mata, A.K. Gupta, S.R. Sadras, Biological activities of green silver nanoparticles synthesized with Acorous calamus rhizome extract, European journal of medicinal chemistry, 85 (2014) 784-794.

[29] D.S. Balaji, S. Basavaraja, R. Deshpande, D.B. Mahesh, B.K. Prabhakar, A. Venkataraman, Extracellular biosynthesis of functionalized silver nanoparticles by strains of Cladosporium cladosporioides fungus, Colloids Surf B Biointerfaces, 68 (2009) 8892.

[30] P. Mukherjee, A. Ahmad, D. Mandal, S. Senapati, S.R. Sainkar, M.I. Khan, R. Ramani, R. Parischa, P.V. Ajayakumar, M. Alam, M. Sastry, R. Kumar, Bioreduction of aucl(4)(-) ions by the fungus, verticillium sp. and surface trapping of the gold nanoparticles formed, Angew Chem Int Ed Engl, 40 (2001) 3585-3588.

[31] S. Iravani, B. Zolfaghari, Green synthesis of silver nanoparticles using Pinus eldarica bark extract, Biomed Res Int, 2013 (2013) 639725.

[32] M. Sathishkumar, K. Sneha, S.W. Won, C.W. Cho, S. Kim, Y.S. Yun, Cinnamon zeylanicum bark extract and powder mediated green synthesis of nano-crystalline silver particles and its bactericidal activity, Colloids Surf B Biointerfaces, 73 (2009) 332-338. 
[33] S.L. Smitha, K.M. Nissamudeen, D. Philip, K.G. Gopchandran, Studies on surface plasmon resonance and photoluminescence of silver nanoparticles, Spectrochim Acta A Mol Biomol Spectrosc, 71 (2008) 186-190.

[34] A. Cornish-Bowden, Fundamentals of enzyme kinetics, 4th ed., Wiley-Blackwell, Weinheim, Germany, 2012.

[35] V.R. Pasupuleti, Prasad, R.A. Shiekh, S.K. Balam, G. Narasimhulu, C.S. Reddy, I. Ab Rahman, S.H. Gan, Biogenic silver nanoparticles using Rhinacanthus nasutus leaf extract: synthesis, spectral analysis, and antimicrobial studies, Int J Nanomedicine, 8 (2013) 3355-3364.

[36] A. Bankar, B. Joshi, A.R. Kumar, S. Zinjarde, Banana peel extract mediated synthesis of gold nanoparticles, Colloids Surf B Biointerfaces, 80 (2010) 45-50.

[37] T. Prathna, N. Chandrasekaran, A.M. Raichur, A. Mukherjee, Biomimetic synthesis of silver nanoparticles by Citrus limon (lemon) aqueous extract and theoretical prediction of particle size, Colloids and Surfaces B: Biointerfaces, 82 (2011) 152-159. 


\section{Figure Legends}

Fig 1. Synthesis of AgNPs using Artemisia absinthium extract. (a) Eppendorf tubes showing reaction mixtures of Artemisia absinthium aqueous extract and silver nitrate $\left(\mathrm{AgNO}_{3}\right)$ in different ratios indicated on the top $(v / v)$. Extract and silver nitrate solutions alone are indicated by a '+' sign. (b) UV-vis absorbance profiles (300-600 nm) of the above reaction mixtures after 24 hours of incubation.

Fig 2. Synthesis kinetics of AgNPs. Reaction mixtures consisted of A. absinthium aqueous extract and silver nitrate $\left(\mathrm{AgNO}_{3}\right)$ mixed in a 1:1 $(v / v)$ ratio. (a) UV-vis spectrum profiles are shown for the indicated time points after the start of reaction. (b) Scatter plot displaying AgNPs yield (measured as the sum of $\lambda$ 400-500 nm) vs. reaction incubation time. Curve represents fit of the data to exponential model.

Fig 3. Titration kinetics of AgNPs synthesis: AgNPs synthesis in response to: (a) different concentrations of $\mathrm{AgNO}_{3}$ at the constant $\left(10 \mathrm{mg} \mathrm{mL}^{-1}\right)$ plant extract concentration, (c) different concentrations of plant aqueous extract at constant $\mathrm{AgNO}_{3}$ concentrations of $1.0 \mathrm{mM}$ (e) $2.0 \mathrm{mM} \mathrm{AgNO}_{3}$, and (g) Plant extract $\left(10 \mathrm{mg} \mathrm{mL}^{-1}\right)$ and $\mathrm{AgNO}_{3}(2 \mathrm{mM})$ mixed as; from $100 \mu \mathrm{L}: 900 \mu \mathrm{L}(1: 9 \nu / v)$ to $900 \mu \mathrm{L}: 100 \mu \mathrm{L}(9: 1 \nu / v)$, respectively, on the $x$-axis. $(\mathbf{b}, \mathbf{d}, \mathbf{f}, \mathbf{h})$ Corresponding silver nitrate conversion efficiencies [(weight of input $\mathrm{Ag}^{+} /$weight of output AgNPs)x100] of the above reactions are shown in the panels on the right side. Y2-axes in panels a, c, e and g are AgNP quantities in $\mu \mathrm{g} \mathrm{mL}^{-1}$, whereas Y1-axes show the sum of $\lambda 400-500 \mathrm{~nm}$, which corresponds to AgNP quantity measured in arbitrary optical units. 
Fig 4. Physical characterization of AgNPs. Dynamic Light Scattering (DLS)-based size distribution (left panel) and $\zeta$-potential (right panel) of AgNPs synthesized after reaction of $A$. absinthium extract and silver nitrate mixed in the following ratios: (a) 1:9 (b) 2:8 (c) $3: 7$ (d) $4: 6$ (e) $5: 5$ (f) $6: 4$ (g) $7: 3$ (h) $8: 2$.

Fig 5. Transmission electron microscopy and EDX analysis of AgNPs. (a, b) Transmission electron microscopy (TEM) images of AgNPs synthesized with $A$. absinthium extract and $\mathrm{AgNO}_{3}(2 \mathrm{mM})$ mixed in a 1:1 $(\mathrm{v} / \mathrm{v})$ ratio. Inset shows characteristic lattice fringes and fringe width $(0.23 \mathrm{~nm}$, indicated with arrows $)$ of Ag. (c) EDX spectra of AgNPs showing the characteristic peak for Ag. Peaks of N, C and Cu, which result from carbon-coated copper grids and biological specimens are also shown. 
Table 1: Synthesis formulation and the average sizes of synthesized silver nanoparticles (AgNPs)

\begin{tabular}{lll}
\hline $\begin{array}{l}\text { Plant extract }(\mathbf{1 0} \mathbf{~ m g} \\
\left.\mathbf{m L}^{-\mathbf{1}}\right)+\mathbf{A g N O}_{\mathbf{3}} \\
(\mathbf{2} \mathbf{m} \mathbf{M})\end{array}$ & $\begin{array}{l}\text { Plant extract : } \\
\text { AgNO }_{3} \text { ratios }(\boldsymbol{v} / \boldsymbol{v})\end{array}$ & $\begin{array}{l}\text { AgNPs } \\
\text { average size } \\
(\mathbf{r . n m})\end{array}$ \\
\hline $100 \mu 1+900 \mu 1$ & $1: 9$ & 40.03 \\
$200 \mu 1+800 \mu 1$ & $2: 8$ & 39.12 \\
$300 \mu 1+700 \mu 1$ & $3: 7$ & 39.71 \\
$400 \mu 1+600 \mu 1$ & $4: 6$ & 46.90 \\
$500 \mu 1+500 \mu 1$ & $5: 5$ & 51.70 \\
$400 \mu 1+600 \mu 1$ & $6: 4$ & 76.92 \\
$300 \mu 1+700 \mu 1$ & $7: 3$ & 71.57 \\
$200 \mu 1+800 \mu 1$ & $8: 2$ & 80.34 \\
\hline
\end{tabular}


a
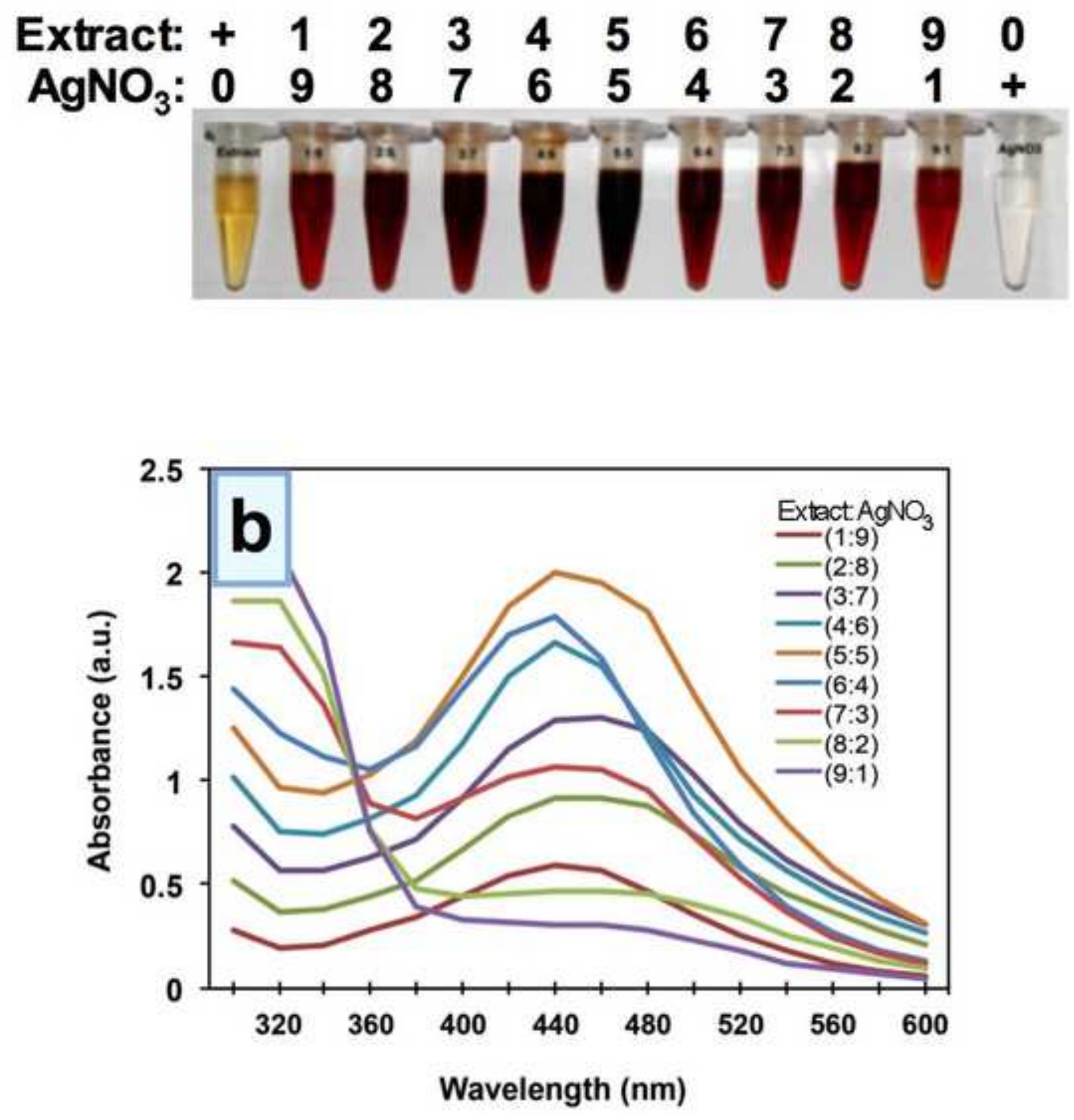
Figure 2

Click here to download high resolution image
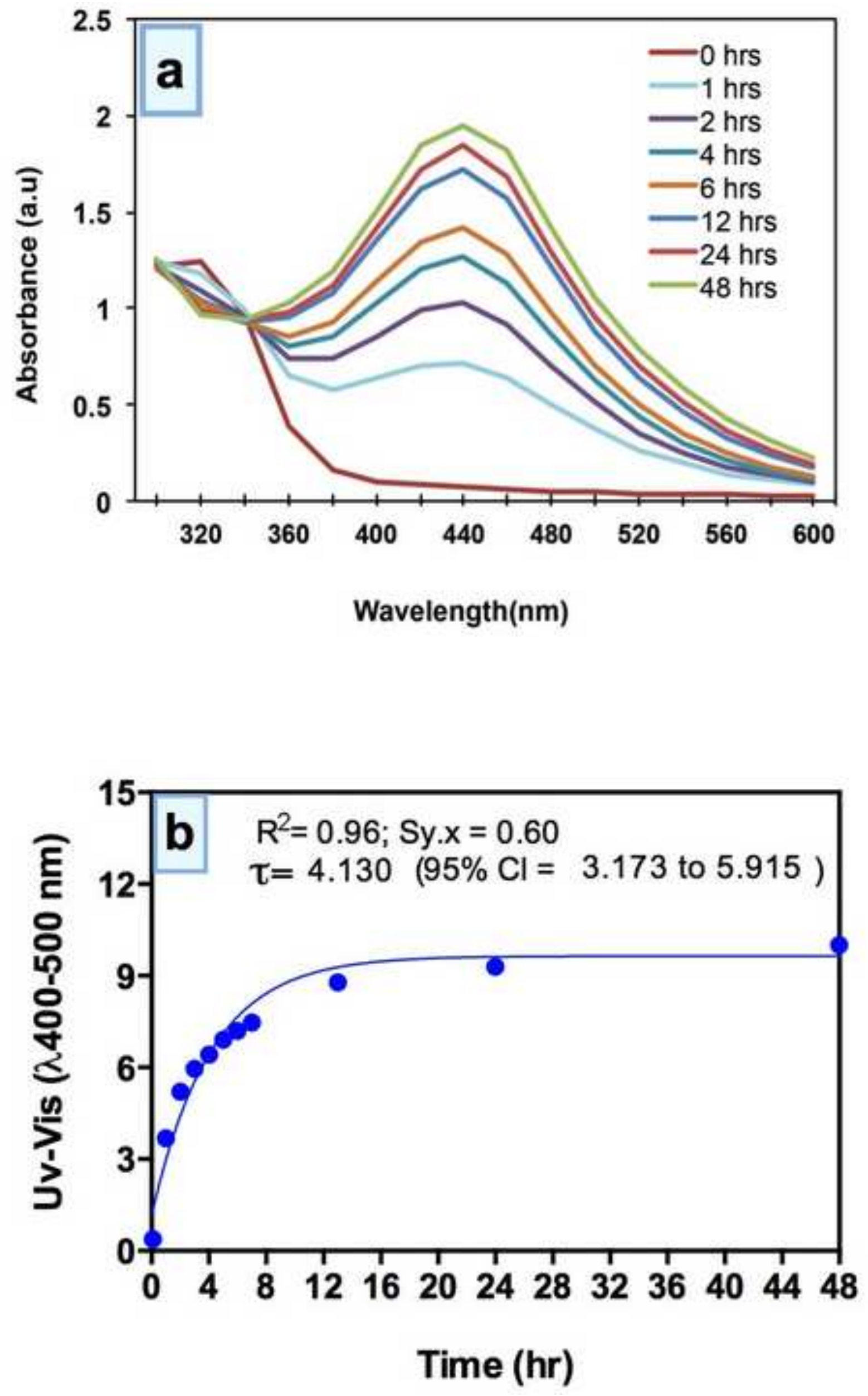


\section{Figure 3}

Click here to download high resolution image
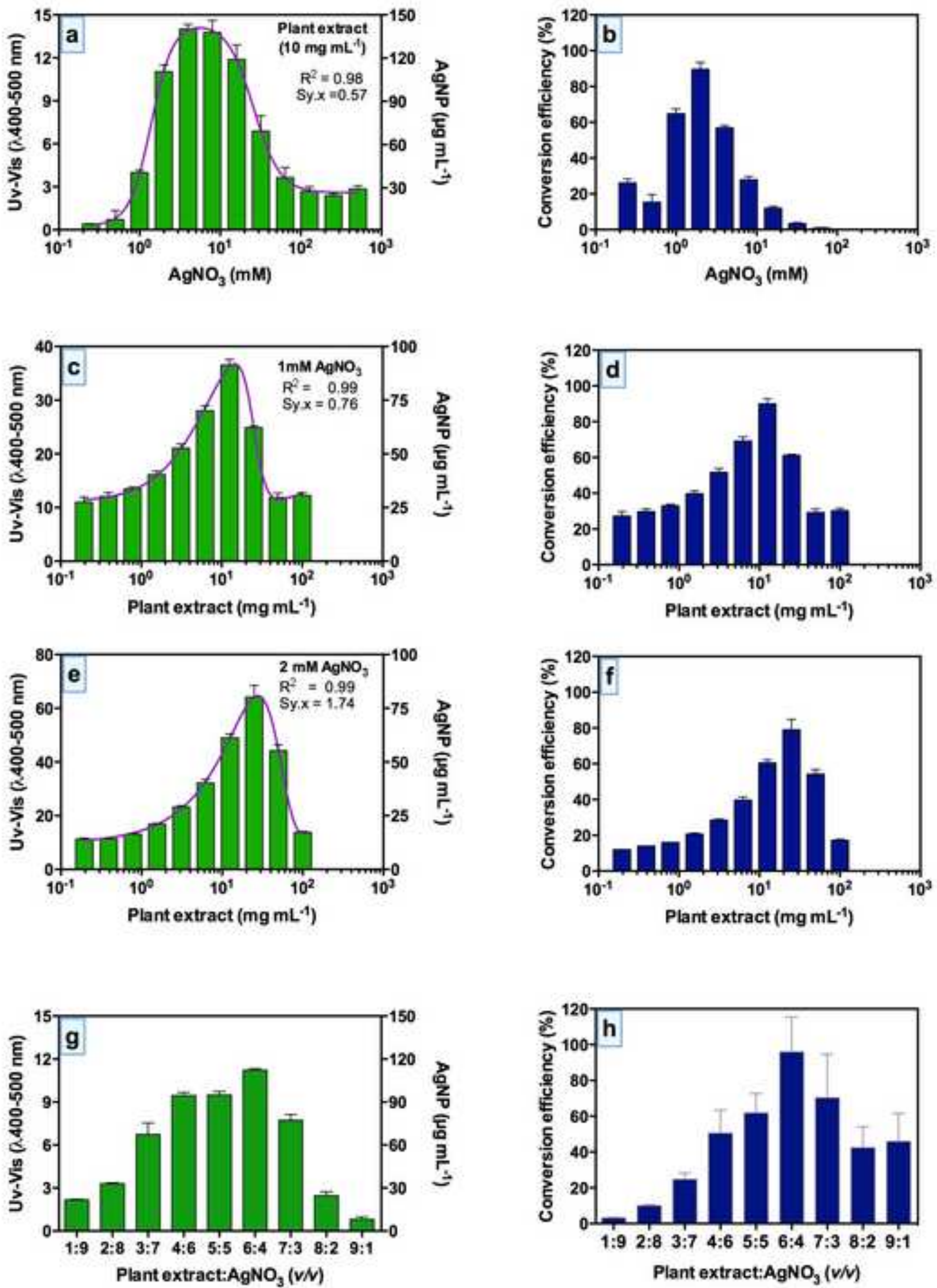


\section{Size (radius, $\mathrm{nm}$ )}
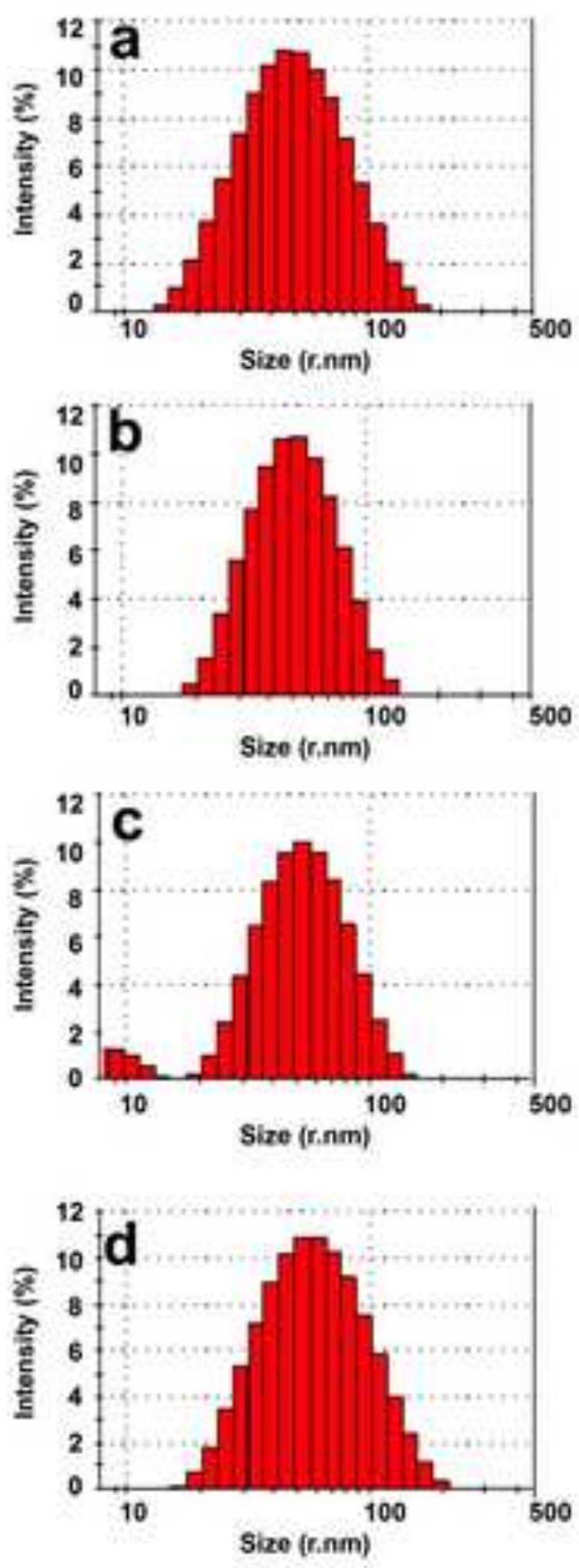
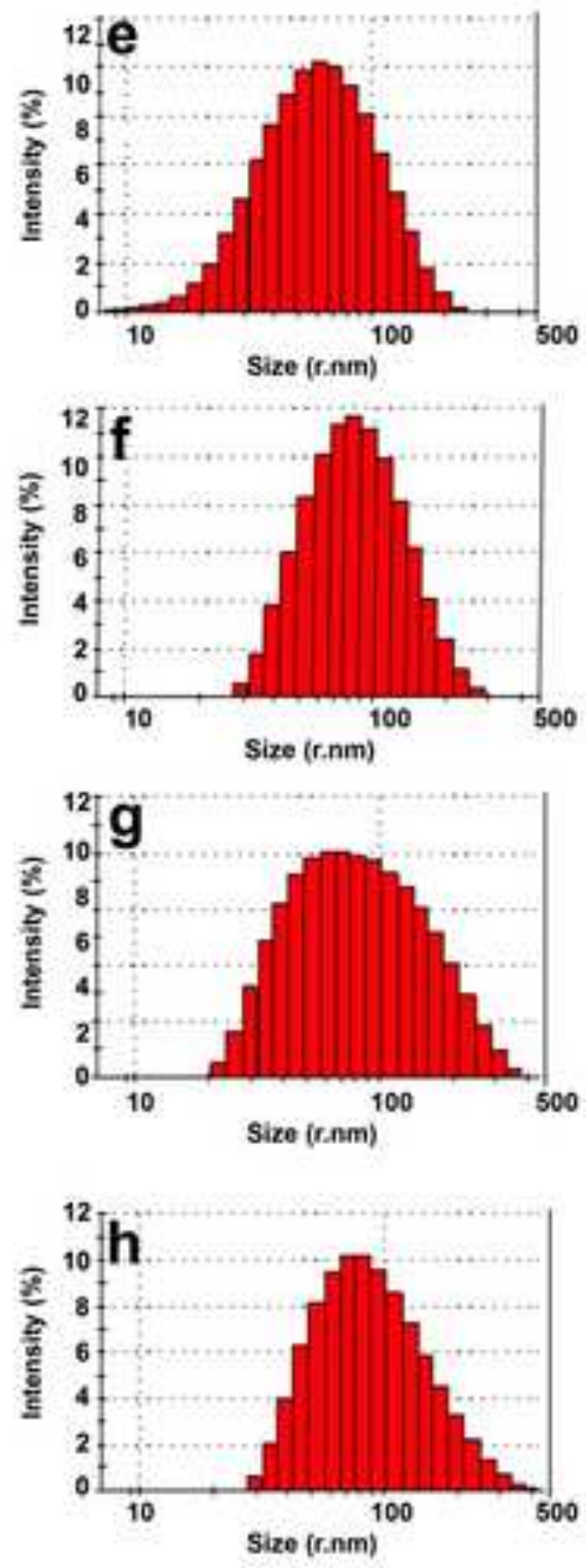

\section{Zeta potential}
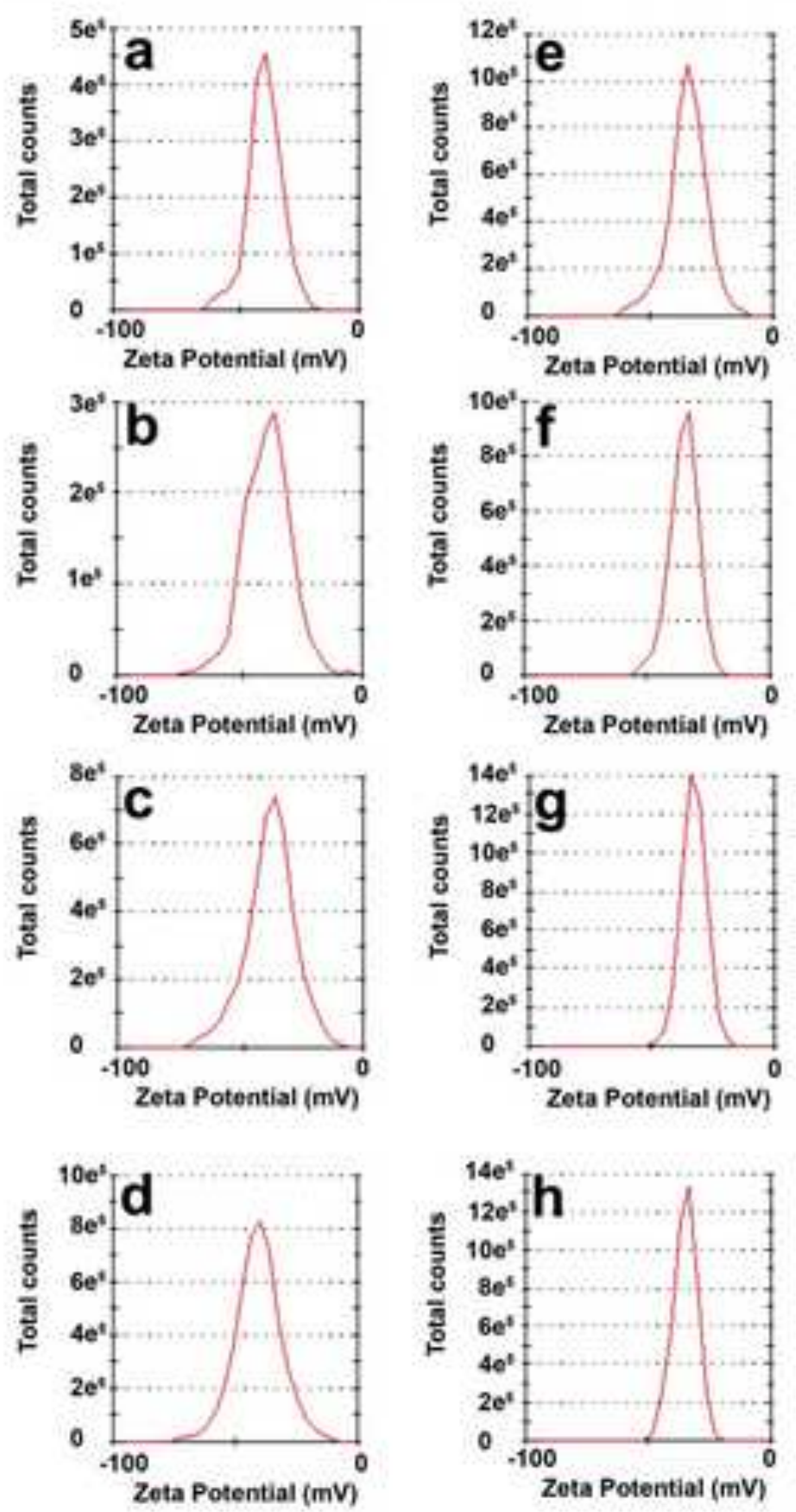
Click here to download high resolution image
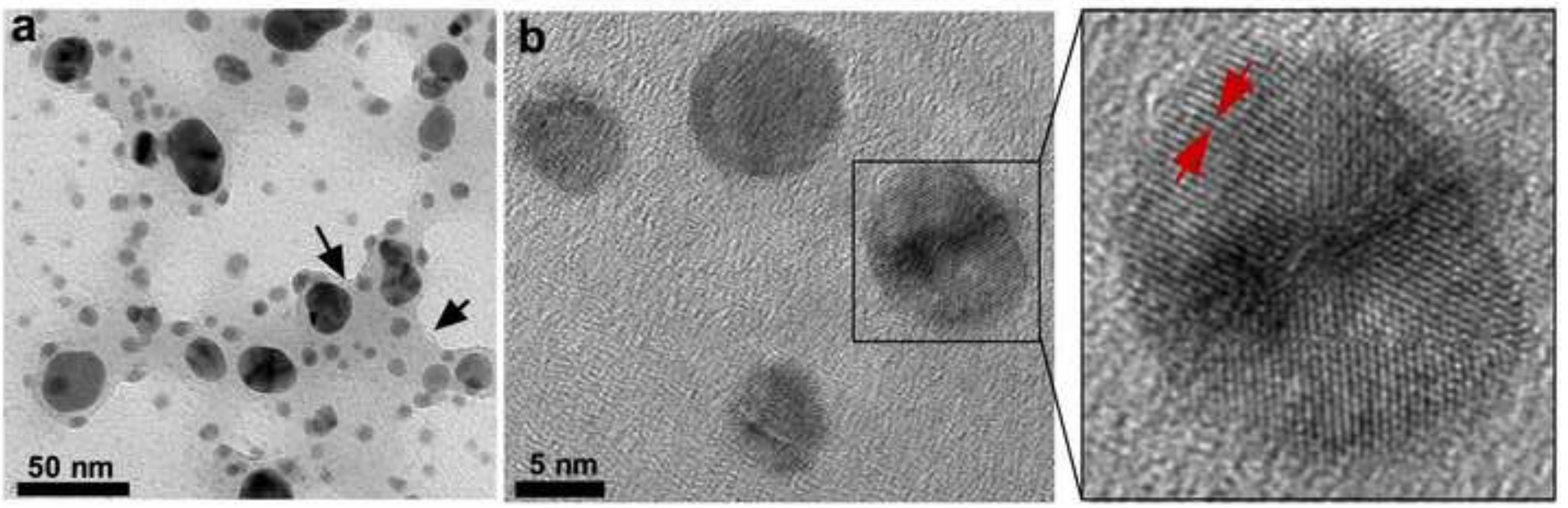

C

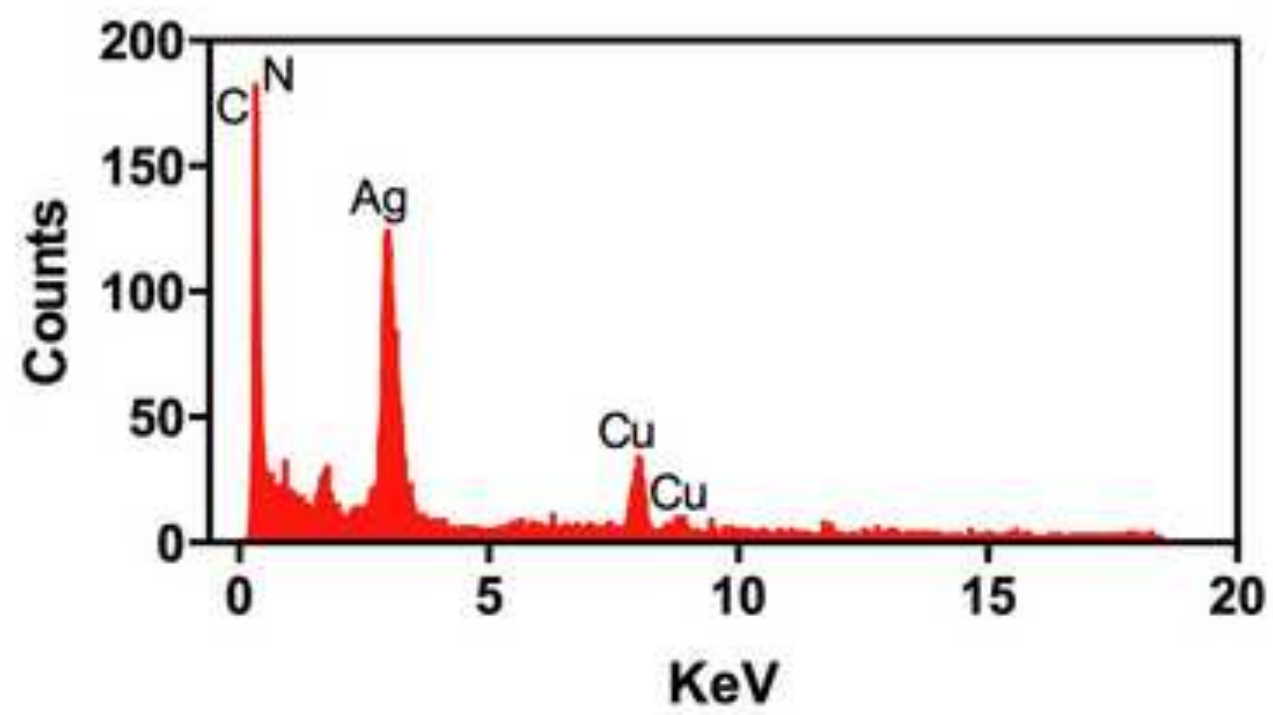

